VOL. 38 (1988) [239-253]

\title{
ON MOSCO CONVERGENCE OF CONVEX SETS
}

\author{
Gerald Beer
}

\begin{abstract}
We present a natural topology compatible with the Mosco convergence of sequences of closed convex sets in a reflexive space, and characterise the topology in terms of the continuity of the distance between convex sets and fixed weakly compact ones. When the space is separable, the topology is Polish. As an application, we show that in this context, most closed convex sets are almost Chebyshev, a result that fails for the stronger Hausdorff metric topology.
\end{abstract}

\section{INTRODUCTION}

About twenty years ago Mosco [28] introduced a fundamental notation of convergence for sequences of closed convex sets in a reflexive space, now called Mosco convergence, widely applicable to convex optimisation, the solution of variational inequalities, and to the theory of optimal control. Specifically, a sequence $\left\langle C_{n}\right\rangle$ of closed convex sets in a reflexive space $X$ is declared Mosco convergent to a closed convex set $C$ provided:

(i) at each $x$ in $C$ there exists a sequence $\left\langle x_{n}\right\rangle$ convergent strongly to $x$ such that for each $n, x_{n} \in C_{n}^{\prime}$, and

(ii) whenever $\langle n(k)\rangle$ is an increasing sequence of positive integers and $x_{n(k)} \in$ $C_{n(k)}$ for each $k$, then the weak convergence of $\left\langle x_{n(k)}\right\rangle$ to $x$ implies $x \in C$.

The most celebrated result on Mosco convergence is concerned with the convergence of sequences of convex functions (as identified with their epigraphs) on a reflexive space. A sequence of lower semicontinuous proper convex functions $\left\langle f_{n}\right\rangle$ on $X$ is declared Mosco convergent to a convex function $f$ provided $\left\langle\right.$ epi $f_{n}$ 〉 converges in the above sense to epi $f$. Locally, this means at each $x$ in $X$ :

(i) there exists a sequence $\left\langle x_{n}\right\rangle$ convergent strongly to $x$ for which $\lim f_{n}\left(x_{n}\right)=f(x)$, and

(ii) whenever $\left\langle x_{n}\right\rangle$ converges weakly to $x$, then $\liminf f_{n}\left(x_{n}\right) \geqslant f(x)[28$, Lemma 1.10]

Received 18 November 1987

Copyright Clearance Centre, Inc. Serial-fee code: 0004-9729/88 \$A2.00+0.00. 
With this notion of convergence, the Young-Fenchel transform, that is, the conjugate operator, is "continuous": if $\left\langle f_{n}\right\rangle$ is Mosco convergent to $f$, then $\left\langle f_{n}^{*}\right\rangle$ is Mosco convergent to $f^{*}([\mathbf{2 2}, \mathbf{2 9}])$.

More recently, Mosco convergence has reared its head at the foundations of functional analysis [7]. If $X$ is reflexive, then the norm convergence of a sequence $\left\langle y_{n}\right\rangle$ in $X^{*}$ to a nonzero limit $y \in X^{*}$ is equivalent to the Mosco convergence of the sequence of level sets $\left\langle\left\{x \in X \mid\left\langle y_{n}, x\right\rangle=\alpha\right\}\right\rangle$ to $\{x \in X:\langle y, x\rangle=\alpha\}$, for each real $\alpha$. This is somewhat surprising, for Mosco convergence may be described without reference to norms or distance at all.

In his monograph on variational convergence [2], Attouch displayed a topology compatible with Mosco convergence of convex functions, and showed that the topology is Polish (second countable and completely metrisable) when the reflexive space is, in addition, separable. An induced topology on convex sets is then obtained, identifying a set with its indicator function (Mosco convergence of a sequence of closed convex sets is equivalent to the Mosco convergence of their indicator functions). The approach of Attouch is highly indirect, resting on the equivalence between Mosco convergence of sequences of functions and the pointwise convergence of their Moreau-Yosida approximates, under a suitable renorming of the space.

The purpose of this article is provide a direct and much simpler description of the topology of Mosco convergence for convex sets, that seems more tractible for handling questions of a geometrical nature. As a particular application, we show that with respect to this topology, most convex sets in a separable reflexive space are almost Chebyshev, a result that fails when the stronger Hausdorff metric topology is used instead.

At this time, the most complete reference on Mosco convergence of sets is the thesis of Sonntag [32]. The reader may also consult $[2,30,34]$ and of course, the papers of Mosco himself.

\section{NOTATION AND TERMINOLOGY}

In the sequel, all spaces $X$ are real normed linear spaces. We distinguish the following classes of subsets of $X$;

$$
\begin{gathered}
C(X)=\text { the closed convex nonempty subsets of } X \\
K(X)=\text { the weakly compact nonempty subsets of } X \\
O(X)=\text { the strongly open nonempty subsets of } X \\
C L(X)=\text { the strongly closed nonempty subsets of } X
\end{gathered}
$$

We denote the closed unit ball of $X$ by $U$ and the origin of $X$ by $\theta$. If $\left\{x, x_{1}, x_{2}, \ldots\right\} \subseteq X$ and $\left\langle x_{n}\right\rangle$ converges strongly (respectively weakly) to $x$, we write $x=\lim x_{n}\left(\right.$ respectively $\left.x=w-\lim x_{n}\right)$. If $A \in C L(X)$ and if $B \in C L(X)$, we set 
$d(A, B)=\inf \{\|a-b\| \mid a \in A, b \in B\}$, forgiving the abuse $d(x, A)$ for $d(\{x\}, A)$. If $X$ is reflexive and $x \in X$ and $C \in C(X)$, then $x$ has at least one nearest point in $C$. The set of nearest points to $x$ in $C$ is called the metric projection of $x$ onto $C$, and will be denoted by $P(x, C)$. A set $C$ is called Chebyshev [20] (respectively almost Chebyshev [19]) if $\{x \in X \mid P(x, C)$ is a singleton $\}$ is all of $X$ (respectively is a dense and $G_{\delta}$ subset of $\left.X\right)$. If $X$ is strictly convex, then each element of $C(X)$ is Chebyshev.

For background, it is perhaps worthwlile to list the basic topologies on $C(X)$. All of them are considered (in a more general setting) either explicitly or implicitly in a recent unifying paper of Francaviglia, Levi, and Lechicki [18]. If $A \in C(X)$ and $B \in C(X)$, then the Hausdorff distance $H$ between the two sets is given by

$$
H(A, B)=\inf \{\alpha \mid A+\alpha U \supseteq B \text { and } B+\alpha U \supseteq A\} .
$$

Hausdorff distance so defined yields an infinite valued complete metric on $C(X)([\boldsymbol{\theta}$, 24]). We denote the associated topology by $\tau_{H}$. It is well-known that Hausdorff metric convergence of a net of sets is equivalent to the uniform convergence of their distance functions; more precisely,

$$
H(A, B)=\|d(\cdot, A)-d(\cdot, B)\|_{\infty} .
$$

A therefore weaker notion of convergence for net of sets is pointwise convergence of their distance functions. It is known that this convergence is compatible with a completely regular topology $\tau_{W}$ on $C(X)$, called the Wijsman topology, which is metrisable when $X$ is separable $([\mathbf{1 8}, \mathbf{2 6}])$.

We now turn to the standard "hit and miss" topologies in the literature. For each nonempty subset $E$ of $X$, we define collections of closed convex sets $E^{+}$and $E^{-}$by the formulas

$$
E^{+}=\{C \in C(X) \mid C \subseteq E\} \quad \text { and } \quad E^{-}=\{C \in C(X) \mid C \cap E \neq \emptyset\}
$$

The topologist's favourite topology of this genre is the finite or Vietoris topology ([24, 27]) $\tau_{V}$, which includes as a subbase all sets of the form $V^{-}$and $V^{+}$where $V \in O(X)$. Thus, a basic open neighbourhood of a convex set $C$ consists of all convex sets that hit each member of a prescribed finite list of open sets, and which miss (fail to hit) a prescribed closed set. For the purposes of analysis, this topology is extremely pathological. The Fell topology $\tau_{F}[\mathbf{1 6}]$, also called the the topology of closed convergence [24] or the topology of set convergence [2], has as a subbase all sets of the form $V^{-}$where $V \in O(X)$ and $\left(K^{c}\right)^{+}$where $K$ is (strongly) compact. The Fell topology seems to be well-behaved only when $X$ is finite dimensional; in this case it is Polish (this follows 
from [24, Theorem 4.5.5] and the fact that a $G_{\delta}$ subset of a completely metrisable space is completely metrisable; see more directly the comments preceding Lemma 4.1 of [6]). By the ball topology $\tau_{B}[4]$, we mean the topology with a subbase consisting of all sets of the form $V^{-}$where $V \in O(X)$ and $\left(B^{\mathrm{c}}\right)^{+}$where $B$ is closed ball. We note that $O(X)$ may be replaced by the collection of open balls in the description of a subbase for $\tau_{B}$, justifying the terminology. It is well known that $\tau_{F}=\tau_{W}=\tau_{B}$ when $X$ is finite dimensional (see, for example, Theorem 2.3 of [4]).

Finally, we may consider convergence of convex sets to mean pointwise convergence of their support functions $([\mathbf{1 2}, \mathbf{3 1}])$. In finite dimensions, this is neither stronger nor weaker than convergence with respect to the Fell $(=$ Wijsman $=$ ball $)$ topology $($ see, for example, $[31$, p.29].

\section{The Mosco Topology}

Definition: Let $X$ be a Banach space. The Mosco topology $\tau_{M}$ on $C(X)$ is the topology generated by all sets of the form $V^{-}$where $V \in O(X)$ and $\left(K^{c}\right)^{+}$with $K \in K(X)$.

It is easy to see that the Mosco topology is just the supremum of the Fell topologies on $C(X)$ induced by the strong and weak topologies on $X$ (thus the Mosco topology coincides with the Fell topology if and only if $X$ is finite dimensional). We first show that the Mosco topology is worthy of its name.

Theorem 3.1. Let $X$ be a Banach space, and let $C, C_{1}, C_{2}, C_{3}, \ldots$ be a sequence of closed nonempty convex subsets of $X$. Then $\left\langle C_{n}\right\rangle$ is Mosco convergent to $C$ if and only if $\left\langle C_{n}\right\rangle$ is $\tau_{M}$-convergent to $C$.

Proof: Suppose $\left\langle C_{n}\right\rangle$ is Mosco convergent to $C$. It suffices to show:

(i) if $C \in V^{-}$with $V$ open, then $C_{n} \in V^{-}$eventually, and

(ii) if $C_{n} \in K^{-}$for infinitely many $n$ with $K$ weakly compact, then $C \in K^{-}$.

To prove (i), suppose $x \in C \cap V$; since there exists a sequence $\left\langle x_{n}\right\rangle$ strongly convergent to $x$ with $x_{n} \in C_{n}$ for each $n$, we see that $C_{n}$ meets $V$ eventually.

To prove (ii), let $n(1), n(2), \ldots$ be an increasing sequence of positive integers such that for each $k, C_{n(k)}$ meets $K$. Choose $x_{n(k)}$ in the intersection; by Eberlein's Theorem [33, p.178], $\left\langle x_{n(k)}\right\rangle$ has a subsequence weakly convergent to a point of $K$. But by condition (ii) in the definition of Mosco convergence, we have $x \in C$. Thus, $C \in K^{-}$.

Conversely, suppose $\left\langle C_{n}\right\rangle$ is $\tau_{M}$-convergent to $C$. Verification of condition (i) in the definition of Mosco convergence being routine, we turn to condition (ii). Suppose $x=w-\lim x_{n(k)}$ where $x_{n(k)} \in C_{n(k)}$ for each $k \in Z^{+}$. If $x \notin C$ held, then we could strongly separate $x$ from $C$ by a continuous linear functional, whence by weak 
convergence, there exists an index $k_{0}$ such that for each $k \geqslant k_{0}, x_{n(k)} \notin C$. As a result, $\left\langle C_{n}\right\rangle$ meets the weakly compact set $\{x\} \cup\left\{x_{n(k)} \mid k \geqslant k_{0}\right\}$ frequently, whereas $C$ misses the set. This is incompatible with the $\tau_{M}$-convergence of $\left\langle C_{n}\right\rangle$ to $C$. Thus, $x \in C$, and condition (ii) in the definition of Mosco convergence is verified.

We now characterise the Mosco topology as a weak topology. We need the following well-known facts, which follow immediately from the weak compactness of closed balls in a reflexive space and the weak lower semicontinuity of the norm.

Lemma 3.2. Let $X$ be reflexive space, let $C \in C(X)$ and let $K \in K(X)$. Then:

(a) there exist $c \in C$ and $x \in K$ with $d(C, K)=\|c-x\|$;

(b) if $K$ and $C$ are disjoint, then $d(C, K)>0$;

(c) for each $\alpha>0$, the parallel body $K+\alpha U$ is weakly compact.

Theorem 3.3. Let $X$ be a reflexive space. The Mosco topology is the weakest topology on $C(X)$ such that for each $K \in K(X), A \rightarrow d(K, A)$ is a continuous functional on $C(X)$.

Proof: We first show each such functional is $\tau_{M}$-continuous; it then follows that each is $\tau$-continuous whenever $\tau_{M} \subseteq \tau$. Fix $K \in K(X)$ and define $\delta: C(X) \rightarrow R$ by $\delta(A)=d(K, A)$. Let $\left\langle C_{\lambda}\right\rangle$ be a net in $C(X) \tau_{M}$-convergent to a closed convex set $C$. Choose by Lemma 3.2 (a) $x \in K$ and $c \in C$ with $\|x-c\|=d(K, C)$. For each $\varepsilon>0,\left\langle C_{\lambda}\right\rangle$ meets $c+\varepsilon U$ eventually; so, $\lim \sup \delta\left(C_{\lambda}\right) \leqslant \delta(C)$. To show that $\delta\left(C^{\prime}\right) \leqslant \liminf \delta\left(C_{\lambda}\right)$, let $\alpha>\liminf \delta\left(C_{\lambda}\right)$ be arbitrary. By Lemma 3.2 (c), $K+\alpha U$ is weakly compact, and since it meets $\left\langle C_{\lambda}\right\rangle$ frequently, it must meet $C$ by the definition of $\tau_{M}$-convergence. As a result, $\delta(C) \leqslant \alpha$.

It remains to show that if each such functional is $\tau$-continuous for a topology $\tau$ on $C(X)$, then $\tau \supset \tau_{M}$. To this end, let $C \in C(X)$ be fixed and let $\left\langle C_{\lambda}\right\rangle$ be a net in $C(X) \tau$-convergent to $C$. We show that the net is $\tau_{M}$-convergent to $C$. First, suppose $C \in V^{-}$where $V$ is open. Pick $c \in C$ and $\varepsilon>0$ such that $c+\varepsilon U \subseteq V$; by the $\tau$-continuity of $A \rightarrow d(c, A)$ at $A=C$, for all $\lambda$ sufficiently large, we have

$$
d\left(c, C_{\lambda}\right)=\left|d\left(c, C_{\lambda}\right)-d(c, C)\right|<\varepsilon .
$$

But this means that, eventually, $C_{\lambda} \in V^{-}$. Now suppose $C \in\left(K^{c}\right)^{+}$where $K^{r} \in$ $K(X)$. By Lemma $3.2(\mathrm{~b}), d(K, C)>0$; so, again by $\tau$-continuity of $A \rightarrow d(K, A)$, we have, eventually, $d\left(K, C_{\lambda}\right)>0$. This means that, eventually, $C_{\lambda} \in\left(K^{c}\right)^{+}$.

Theorem 3.4. Let $X$ be reflexive. Then the Mosco topology on $C(X)$ is Hausdorff and completely regular.

Proof: Let $A$ and $B$ be distinct members of $C(X)$. Without loss of generality, we may assume that $A \cap B^{c}$ is nonempty. Pick $a \in A \cap B^{c}$ and let $V=\{x \mid$ 
$\|x-a\|<(.5) d(a, B)\}$ and $K=\{x \mid\|x-a\| \leqslant(.5) d(a, B)\}$. Then $V^{-}$and $\left(K^{-c}\right)^{+}$ are disjoint $\tau_{M}$-neighbourhoods of $A$ and $B$, respectively. For complete regularity, we use Theorem 3.3. Fix $C \in C(X)$ and let $\Omega$ be a $\tau_{M}$-neighbourhood of $C$. Since $\left(E^{c}\right)^{+} \cap\left(F^{c}\right)^{+}=\left((E \cup F)^{c}\right)^{+}$, there exists $\left\{V_{1}, V_{2}, \ldots, V_{n}\right\} \subseteq O(X)$ and $K \in K(X)$ such that

$$
C \in \bigcap_{i=1}^{n} V_{i}^{-} \cap\left(K^{c}\right)^{+} \subseteq \Omega
$$

Without loss of generality, we may assume that $\left\{V_{1}, V_{2}, \ldots, V_{n}, K\right\}$ are pairwise disjoint. Choose $c_{i} \in C \cap V_{i}$, for $i=1, \ldots, n$. By Lemma $3.2(\mathrm{~b}), d(C, K)>0$; so there exists $\alpha>0$ such that $(K+\alpha U) \cap C=\emptyset$ and for each $i, c_{i}+\alpha U \subset V_{i}$. For each $i \in\{1,2, \ldots, n\}$ define $g_{i}:\left\langle C(X), \tau_{M}\right\rangle \rightarrow R$ by $g_{i}(A)=\max \left\{0,1-\alpha^{-1} d\left(c_{i}, A\right)\right\}$, and define $h:\left\langle C(X), \tau_{M}\right\rangle \rightarrow R$ by $h(A)=\min \left\{1, \alpha^{-1} d(K, A)\right\}$. By Theorem 3.3, all of these functions are $\tau_{M}$-continuous, and their product maps $C$ to one, $C(X)-\Omega$ to zero, and $C(X)$ to $[0,1]$.

We now compare $\tau_{M}$ with the topologies mentioned in the introduction.

Theorem 3.5. Let $X$ be reflexive. On $C(X)$, we have:

(a) $\tau_{F} \subseteq \tau_{W}=\tau_{B} \subseteq \tau_{M}$;

(b) $\tau_{M} \subseteq \tau_{H}$;

(c) $\tau_{M} \subseteq \tau_{V}$.

Proof: (a). The inclusion $\tau_{F} \subseteq \tau_{W} \subseteq \tau_{B}$ without reflexivity or convexity are known ([18, Propositions 2.1 and 2.3$]$ and $\left[4\right.$, p.84]), and the inclusion $\tau_{B} \subseteq \tau_{M}$ is obvious, because closed balls are weakly compact. It remains to prove that $\tau_{B} \subseteq$ $\tau_{W}$. Suppose $\left\langle C_{\lambda}\right\rangle$ is Wijsman convergent to $C$. Then for each $p$ in $X$, we have $\limsup d\left(p, C_{\lambda}\right) \leqslant d(p, C)$, which is equivalent to saying that whenever $C$ meets an open set $V$, then $\left\langle C_{\lambda}\right\rangle$ meets $V$ eventually. It remains to show that if $C$ fails to meet a ball $p+\alpha U$, then $\left\langle C_{\lambda}\right\rangle$ fails to meet the ball eventually. By Lemma 3.2(b) we have $d(p, C)>\alpha$. By Wijsman convergence, we have $\lim \inf d\left(p, C_{\lambda}\right) \geqslant d(p, C)$; so there exists an index $\lambda_{0}$ such that if $\lambda \geqslant \lambda_{0}$, then $d\left(p, C_{\lambda}\right)>\alpha$. This means that for $\lambda \geqslant \lambda_{0}$, we have $C_{\lambda} \in\left((p+\alpha U)^{c}\right)^{+}$.

(b) We show that each subbasic open set in the Mosco topology is $\tau_{H}$-open. Suppose $C \in V^{-}$where $V$ is open. Pick $c \in C$ and $\varepsilon>0$ such that $c+\varepsilon U \subseteq V$. Clearly if $H(C, A)<\varepsilon$, then $A \in V^{-}$. Next suppose that $C \in\left(K^{c}\right)^{+}$where $K$ is weakly compact. By Lemma $3.2(\mathrm{~b}), d(C, K)>0$, and if $H(C, A)<d(C, K)$, then $K \cap A=\emptyset$, that is $A \in\left(K^{c}\right)^{+}$.

(c) This is immediate from $K(X) \subseteq C L(X)$. 
Sequential versions of the inclusions $\tau_{F} \subset \tau_{W}, \tau_{M} \subset \tau_{H}$ and $\tau_{W} \subset \tau_{M}$ for convex sets in a reflexive space can be found in [3] and in [32], and it is well-known that the first two inclusions may be proper (see, for example, [3, Examples 1 and 5] and [32, p.I.32]. On the other hand, that the inclusion $\tau_{W} \subseteq \tau_{M}$ may be proper was only recently discovered by me [7]. We remark that in a nonreflexive space, $\tau_{W}$ and $\tau_{M}$ may in fact be noncomparable (see [3, Example 2] and [32, p.II.25]). We choose not to dwell on pathology here.

ThEOREM 3.6. Let $X$ be reflexive. Then $\left\langle C(X), \tau_{M}\right\rangle$ is path-connected.

Proof: It is known (see, for example, $[5]$ ) that if $A$ and $B$ are bounded elements of $C^{\prime}(X)$, then the straight line path $g:[0,1] \rightarrow\left\langle C(X), \tau_{H}\right\rangle$ defined by

$$
g(\alpha)=\operatorname{cl}(\alpha A+(1-\alpha) B)
$$

is continuous. Thus, the path remains continuous if we replace $\tau_{H}$ by the weaker $\tau_{M}$. Thus, it remains to show that each unbounded element $C^{\prime}$ of $C(X)$ can be joined by $\tau_{M}$-continuous path to some bounded element of $C(X)$. We may assume without loss of generality that $C$ contains the origin $\theta$, for if $c \in C$ and $E=C-c$, then it easy to check that $f:[0,1] \rightarrow\left\langle C(X), \tau_{M}\right\rangle$ defined by $f(\alpha)=\alpha c+E$ is a continuous path from $E$ to $C$.

Assuming now that $\theta \in C$, we produce a $\tau_{M}$-continuous path from $C \cap U$ to $C$. Define $h:[0,1] \rightarrow\left\langle C(X), \tau_{M}\right\rangle$ by

$$
H(\alpha)= \begin{cases}{\left[(1-\alpha)^{-1} U\right] \cap C} & \text { if } \alpha<1 \\ C & \text { if } \alpha=1\end{cases}
$$

We show that the inverse image of each subbasic open set is open. First suppose that $V$ is open in $X$ and $h\left(\alpha_{0}\right) \in V^{-}$. Since $h$ is increasing with respect to set inclusion, if $\alpha>\alpha_{0}$, then $h(\alpha) \in V^{-}$. Thus, if $\alpha_{0}=0$, then $h(\alpha) \in V^{-}$for all $\alpha$. If $\alpha_{0}=1$, then for some $\alpha_{1}<1$, we have $h\left(\alpha_{1}\right) \in V^{-}$, whence $h(\alpha) \in V^{-}$for all larger $\alpha$. The possibility $0<\alpha_{0}<1$ remains. Choose $c \in h\left(\alpha_{0}\right) \cap V$. If $c=\theta$, then for all $\alpha$ we have $h(\alpha) \in V^{-}$. Otherwise, since $V$ is open and $\theta \in C$ there exists $\beta \in(0,1)$ for which $\beta c \in C \cap V$. Now $\beta\|c\|<\|c\| \leqslant\left(1-\alpha_{0}\right)^{-1}$, whence $\alpha_{0}>(\beta\|c\|)^{-1}(\beta\|c\|-1)$. If $\alpha>(\beta\|c\|)^{-1}(\beta\|c\|-1)$, then $(1-\alpha)^{-1}>\beta\|c\|$, and we have $\beta c \in h(\alpha) \cap V$. Thus, $h(\alpha) \in V^{-}$, and the inverse image of $V^{-}$is open in all cases.

It is easier to show that the inverse image of $\left(K^{c}\right)^{+}$is open whenever $K$ is weakly compact. Suppose $h\left(\alpha_{0}\right) \in\left(K^{c}\right)^{+}$. Since $h$ is increasing, $h(\alpha) \in\left(K^{c}\right)^{+}$for each $\alpha<\alpha_{0}$. We may thus assume that $\alpha_{0}<1$. It remains to produce $\epsilon>0$ such that whenever $\alpha \in\left(\alpha_{0}, \alpha_{0}+\varepsilon\right)$, then $h(\alpha) \in\left(K^{c}\right)^{+}$. If no such $\varepsilon$ exists, then for each 
$n \in \mathbf{Z}^{+}$, we can find $\alpha_{n} \in\left(\alpha_{0}, \alpha_{0}+1 / n\right)$ with $h\left(\alpha_{n}\right) \in K^{-}$. Now $\left\{h\left(\alpha_{n}\right) \cap K\right.$ । $\left.n \in \mathbf{Z}^{+}\right\}$is a family of weakly compact sets with the finite intersection property, and

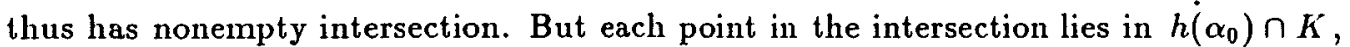
contradicting $h\left(\alpha_{0}\right) \in\left(K^{c}\right)^{+}$. Thus, an appropriate $\varepsilon$ can be found, and the inverse image or $\left(K^{c}\right)^{+}$is open. We conclude that $h$ is a $\tau_{M}$-continuous path from $C \cap U$ to $C$, and the proof of path connectedness of the hyperspace is complete.

\section{4. $\left\langle C(X), \tau_{M}\right\rangle$ is Polish when $X$ is Separable}

Although our approach to the Polish metrisability of Mosco convergence when $X$ is separable and reflexive is somewhat different from that of Attouch, they share a common thread: ultimately, they both depend on the powerful renorming theorem of John and Zizler ([21, 15, p.185]). Recall that a Banach space is called locally uniformly convex if for each $x_{0}$ of norm 1 , whenever $\left\langle x_{n}\right\rangle$ is a sequence of vectors of norm 1 satisfying $\lim _{n \rightarrow \infty}\left\|x_{n}+x_{0}\right\|=2$, then $\lim x_{n}=x_{0}[15]$.

John-Zizler renorming Theorem. Let $X$ be a reflexive Banach space. Then $X$ admits an equivalent norm such that both $X$ and $X^{*}$ (with the dual norm) are locally uniformly convex.

Actually, the result applies to a more inclusive class of spaces, the weakly compactly generated ones. Of curse, the result is a strengthening of the Kadec-Klee renorming theorem for spaces with separable dual. As a first step, we determine when $\left\langle C(X), \tau_{M}\right\rangle$ is first countable.

Lemma 4.1. Let $X$ be a reflexive space. Then $\left\langle C(X), \tau_{M}\right\rangle$ is first countable if and only if $X$ is separable.

Proof: For sufficiency, let $E$ be a countable dense subset of $X$ and let $Q^{+}$be the positive rationals. Evidently, the topology generated by $\left\{V^{-} \mid V \in O(X)\right\}$ is equally well generated by $\left\{(x+\alpha(\text { int } U))^{-} \mid x \in E\right.$ and $\left.\alpha \in \mathbb{Q}^{+}\right\}$. Now fix $C \in C(X)$. It remains to show that the topology generated by $\left\{\left(K^{\mathrm{c}}\right)^{+} \mid K \in K(X)\right\}$ has a countable local base at $C$. Since $X$ is separable, $C^{c}$ is Lindelöf so that the closed convex set $C$ is a countable intersection of closed halfspaces [20, p.7]. From this, there exists a collection of open halfspaces $\left\{H_{i} \mid I \in \mathbb{Z}^{+}\right\}$each containing $C$ whose closures have interesction $C$. By the weak compactness of $K$, there exists a finite subset $F$ of $\mathbb{Z}^{+}$such that $K \subset \cup\left\{H_{i}^{c} \mid i \in F\right\}$. Choose $\alpha \in \mathbb{Q}^{+}$with $K \subseteq \alpha U$. Then $\alpha U \cap\left(\cup\left\{H_{i}^{c} \mid i \in F\right\}\right)$ is a weakly compact set disjoint from $C$ that contains $K$. This shows that all sets of the form

$$
\bigcap_{i \in F}\left[\left(\alpha U \cap H_{i}^{c}\right)^{c}\right]^{+}
$$


where $F \subseteq \mathrm{Z}^{+}$is finite and $\alpha \in \mathbb{Q}^{+}$determine a local base at $C$ for the topology generated by $\left\{\left(K^{c}\right)^{+} \mid K \in K(X)\right\}$.

For necessity, suppose $X$ is nonseparable. We show that first countability of $\tau_{M}$ fails at the closed convex set $C=X$. Suppose to the contrary that $\tau_{M}$ had a countable local base at $C$. Then it would have one of the form $\left\{\Omega(n, k) \mid n \in \mathbf{Z}^{+}\right.$and $\left.k \in \mathbb{Z}^{+}\right\}$ where for each $n$ and $k$,

$$
\Omega(n, k)=\bigcap_{i=1}^{m(n)}\left\{x \mid\left\|x-p_{n i}\right\|<1 / k\right\}^{-}
$$

and $m(n)$ and $\left\{p_{n 1}, p_{n 2}, \ldots, p_{n m(n)}\right\}$ depend on $n$ but not on $k$. Let $W$ be the closure of the subspace generated by $\left\{p_{n i} \mid n \in \mathbb{Z}^{+}\right.$and $\left.i \leqslant m(n)\right\}$. Since $X$ is assumed nonseparable, $W \neq X$, and there exists $p \in X$ with $W \cap(p+U)=\emptyset$. Clearly, $\{x \mid\|x-p\|<1\}^{-}$is a neighbourhood of $C$ containing no $\Omega(n, k)$.

One consequence of Lemma 4.1 is that sequences determine the Mosco topology if and only if $X$ is separable.

LeMmA 4.2. Let $X$ be a separable space. Then $\left\langle C(X), \tau_{M}\right\rangle$ is separable.

Proof: If $E$ is a countable dense subset of $X$, then the polytopes with vertices in $E$ are $\tau_{M}$-dense in $C(X)$.

Since the Mosco topology depends only on the topology of $X$ and not on the particular norm chosen, we are free to renorm the space. If we renorm it so that $X$ and $X^{*}$ are both locally uniformly convex, then Mosco convergence of sequences is equivalent to a number of other properties, as Sonntag [32, p.II.24] has shown. The following result was first obtained by Attouch [1], for Hilbert spaces.

Sonntag-Attouch Theorem. Let $X$ be a reflexive space such that both $X$ and $X^{*}$ are locally uniformly convex. Let $C, C_{1}, C_{2}, \ldots$ be a sequence in $C(X)$. The following are equivalent:

(a) $\left\langle C_{n}\right\rangle$ is Mosco convergent to $C$;

(b) $\left\langle C_{n}\right\rangle$ is Wisjman convergent to $C$, that is, for each $x \in X$, we have $d(x, C)=\lim d\left(x, C_{n}\right)$;

(c) for each $x \in X, P\left(x, C^{\prime}\right)=\lim P\left(x, C_{n}\right)$.

Statement (c) makes sense in that a locally uniformly convex space is strictly convex $[15, \mathrm{p.31}-32]$, so that the metric projection is single-valued. Actually, Sonntag obtained his result with less than local uniform convexity. Precisely, he assumed that both $X$ and $X^{*}$ are strictly convex, and that in both spaces, weak convergence plus convergence of norms forces strong convergence (Holmes [20] calls a space with these 
two properties an E-space). A proof based on Moreau-Yosida approximation can be found in [2]. We note that contition (b), in conjunction with Theorem 3.5, says that in a separable reflexive locally uniformly convex space, we have $\tau_{M}=\tau_{B}=\tau_{W}$. Since $\tau_{W}$ is metrisable when $X$ is separable $([\mathbf{1 8}, \mathbf{2 6}]), \tau_{M}$ is second countable and metrisable when $X$ is separable and reflexive. To establish complete metrisability, we use condition (c), which is formally much stronger than (b).

Theorem 4.3. Let $X$ be a separable reflexive space. Then $\left\langle C(X), \tau_{M}\right\rangle$ is a Polish space.

Proof: By the John-Zizler renorming theorem, we may assume that each element of $C(X)$ is Chebyshev, and the Mosco convergence of sequences in $C(X)$ can be described as in the Sonntag-Attouch Theorem. Let $\left\{x_{k} \mid k \in \mathbb{Z}^{+}\right\}$be a countable dense subset of $X$. For each $k \in \mathbb{Z}^{+}$, we define a pseudometric $\rho_{k}$ on $C^{\prime}(X)$ by $\rho_{k}(A, B)=\min \left\{1,\left\|P\left(x_{k}, A\right)-P\left(x_{k}, B\right)\right\|\right\}$. Now, we write

$$
\rho(A, B)=\sum_{k=1}^{\infty} 2^{-k} \rho_{k}(A, B) .
$$

To see that $\rho$ is a metric on $C(X)$, note that if $A \cap B^{c}$ is nonempty, then there exists $x_{k}$ with $d\left(x_{k}, A\right)<d\left(x_{k}, B\right)$, whence $P\left(x_{k}, A\right) \neq P\left(x_{k}, B\right)$. By Theorem 3.1 and the equivalence of conditions (a) and (c) in the Sonntag-Attouch Theorem, it is clear that $\tau_{M}$-convergence of sequences forces their $\rho$-convergence. On the other hand, $\lim \rho\left(C, C_{n}\right)=0$ ensures that for each $x_{k}$, we have $d\left(x_{k}, C\right)=\lim d\left(x_{k}, C_{n}\right)$; so, by the equicontinuity of distance functions, for each $x \in X$, we have $d(x, C)=\lim d\left(x, C_{n}\right)$. Since $\left\langle C(X), \tau_{M}\right\rangle$ is first countable, $\rho$ is a compatible metric for the hyperspace. It remains to prove completeness.

Let $\left\langle C_{n}^{\prime}\right\rangle$ be a $\rho$-Cauchy sequence in $C(X)$. Then for each $k \in Z^{+}$, the sequence $\left\langle P\left(x_{k}, C_{n}\right)\right\rangle$ is a Cauchy sequence in $X$, which by completeness of $X$, must converge to some point $c_{k}$. Let $C$ be the (norm) closure of $\left\{c_{k} \mid k \in \mathbb{Z}^{+}\right\}$. We first show that $C$ is convex. Let $c_{1} \in C$ and $c_{2} \in C$ be arbitrary. We show that for each $\lambda \in(0,1)$, that $x \equiv \lambda c_{1}+(1-\lambda) c_{2}$ lies in $C$. It suffices to show that for each $\varepsilon>0$, the ball $x+\varepsilon U$ meets $C$. For this, we need only show that $x+(\varepsilon / 4) U$ meets $C_{n}$ eventually, for, if we choose $x_{k}$ in $x+(\varepsilon / 4) U$, then $P\left(x_{k}, C_{n}\right)$ must lie in $x+\varepsilon U$ for all $n$ sufficiently large. By the construction of $C,\left\langle C_{n}\right\rangle$ meets both $c_{1}+(\varepsilon / 4) U$ and $c_{2}+(\varepsilon / 4) U$ eventually. Since each $C_{n}$ is convex, it follows that $\left\langle C_{n}\right\rangle$ also meets $x+(\varepsilon / 4) U$ eventually. This establishes the convexity of $C$.

That $\left\langle C_{n}\right\rangle$ is $\tau_{M}$-convergent to $C$ now follows easily. For each $k \in \mathbf{Z}^{+}$we have

$$
\begin{aligned}
d\left(x_{k}, C\right)=d\left(x_{k},\left\{c_{i} \mid i \in \mathbb{Z}^{+}\right)\right\} & =\lim _{n \rightarrow \infty}\left\|x_{k}-P\left(x_{k}, C_{n}\right)\right\| \\
& =\lim _{n \rightarrow \infty} d\left(x_{k}, C_{n}\right) .
\end{aligned}
$$


By the equicontinuity of distance functions, we have $d(x, C)=\lim d\left(x, C_{n}\right)$ for each $x$ in $X$, and we are done by Theorem 3.1 and the equivalence of conditions (a) and (b) in the Sonntag-Attouch Theorem.

\section{Most Closed convex sets are almost Chebyshev}

By a multifuction $F$ from a topological space $T$ to a topological space $X$, we mean a function from $T$ to $C L(X)$. A multifunction is called upper semicontinuous $([24,25])$ if for each open subset $V$ of $X$, the set $\{t \in T \mid F(t) \subseteq V\}$ is open in $T$. Locally, this means at each $t \in T$, whenever $V$ is a neighbourhood of $F(t)$ and $\left\langle t_{\lambda}\right\rangle$ is a net in $T$ convergent to $t$, then, eventually, $F\left(t_{\lambda}\right) \subseteq V$. If, in addition, the values of $F$ are compact subsets of $X$, we call $F$ an usco map [10]. If $X$ is a Banach space equipped with its weak topology, then an usco map into $X$ so topologised will be called weakly usco.

Let $C$ be a fixed closed convex subset of reflexive space $X$. It is well-known [23] that the metric projection $x \rightarrow P(x, C)$ is weakly usco with respect to the norm topology on $X$ (see, for example, [8] or [13, Theorem 3] for strongly usco counterexamples). Of course, we may view the metric projection as a multifunction with domain $X \times C(X)$. If we equip a reflexive Banach space $X$ with the norm topology and $C(X)$ with $\tau_{M}$, the metric projection remains weakly usco on the product.

Theorem 5.1. Let $X$ be a reflexive Banach space. If $C(X)$ is equipped with the Mosco topology and $X$ has the norm topology, then the metric projection $P$ on $X \times C(X)$ is weakly usco.

PROOF: The values of the metric projection are closed and norm bounded convex sets and thus are weakly compact. Suppose the metric projection fails to be upper semicontinuous at some $\left(x_{0}, C\right)$. Then there exists a weakly open set $W$ containing $P\left(x_{0}, C\right)$ and a net $\left\langle\left(x_{\lambda}, C_{\lambda}\right)\right\rangle_{\lambda \in \Lambda}$ convergent to $\left(x_{0}, C\right)$ such that for each $\lambda$, $P\left(x_{\lambda}, C_{\lambda}\right) \cap W^{c}=\emptyset$. Choose for each $\lambda \in \Lambda$ a point $c_{\lambda}$ in $P\left(x_{\lambda}, C_{\lambda}\right) \cap W^{c}$. By Theorem 3.5, $\left\langle C_{\lambda}^{\prime}\right\rangle$ is Wisjman convergent to $C$, and since $\lim \left\|x_{\lambda}-x_{0}\right\|=0$, it follows that for some $\mu \in \Lambda$, the set $\left\{c_{\lambda} \mid \lambda \geqslant \mu\right\}$ is bounded. Suppose that $\left\{c_{\lambda} \mid \lambda \geqslant \mu\right\} \subseteq \alpha U$. By reflexivity, the ball $\alpha U$ is weakly compact, whence $\left\langle c_{\lambda}\right\rangle$ has a weak cluster point $z$ in $\alpha U$. Suppose now that $z \notin C$. By the separation theorem, there exists $y \in X^{*}$ and $\beta \in \mathbf{R}$ with $\sup \{\langle y, c\rangle \mid c \in C\}<\beta<\langle y, z\rangle$. Consider this weakly compact set: $K^{\circ} \equiv \alpha U \cap\{x \mid\langle y, x\rangle \geqslant \beta\}$. Since $\{x \mid\langle y, x\rangle>\beta\}$ is a weak neighbourhood of $z$, the set $K$ meets $\left\langle C_{\lambda}\right\rangle$ frequently, whereas $K \cap C=\emptyset$. This violates $C=\tau_{M}-\lim C_{\lambda}$, and we conclude that $z \in C$ must hold. By the weak lower semicontinuity of the norm and the Wijsman convergence of $\left\langle C_{\lambda}\right\rangle$ to $C$, it is easy to see that $z \in P\left(x_{0}, C\right)$. On the other hand, each weak cluster point of $\left\langle c_{\lambda}\right\rangle$ must lie in $W^{c}$, contradicting $P\left(x_{0}, C^{\prime}\right) \subset W$. Thus, $P$ is weakly upper semicontinuous at $x_{0}$. 
We note that the metric projection must be weakly usco on $X \times C(X)$, whenever $C(X)$ is equipped with a topology containing the Mosco topology. It turns out that when $X$ is finite dimensional, then the Mosco topology is the weakest such topology $[6] !$

It follows immediately from Theorem 4.3 that when $X$ is separable and reflexive, the space $X \times C(X)$ is a Polish space, provided $C(X)$ is equipped with the Mosco topology. Since the product is a Baire space, the statement the metric projection is single-valued at most points of the product is at least meaningful, in the sense of Baire category. Of course, it also makes sense when $C(X)$ is topologised by Hausdorff distance, but with respect to this topology, the statement may be false, even when $X$ is finite dimensional: if $X=\mathrm{R}^{n}$ is equiipped with the box norm, and if $C$ is a closed halfspace, and $x \notin C$, then $P$ fails to be single-valued in a neighbourhood of $(x, C)$ [11]. We intend to show that the italicised staatement is true for the Mosco topology. The key ingredient is a continuity theorem of Christensen [10], which may be viewed as a variant of the classical Kuratowski-Fort Theorem [17]. We first need a definition.

DEFINITION: Let $T$ be a topological space and let $X$ be a normed linear space. A multifunction $F$ from $T$ to $X$ is called almost lower semicontinuous (a.l.s.c.) at $t$ in $T$ if there exists $x_{0} \in F(t)$ such that for each $\varepsilon>0$, there exists a neighbourhood $V_{\varepsilon}$ of $t$ such that for each $z \in V_{\varepsilon}$, we have $F(z) \cap\left(x_{0}+\varepsilon U\right) \neq \emptyset$.

Although this property was formally considered first by Christensen [10], for compact-valued multifunctions, it agrees with a weaker property introduced by Deutsch and Kenderov that is fundamental in the approximation of convex-valued multifunctions by continuous single-valued functions [14]. Clearly, almost lower semicontinuity is weaker than ordinary lower semicontinuity for multifunctions (see, for example, [24] or $[\mathbf{2 5}])$.

For simplicity, we choose to state a weakened form of the result of Christensen [10]; it actually holds for a much wider class of domains, including the Cech complete spaces.

Christensen's Theorem. Let $T$ be a complete metric space and let $X$ be a Banach space. Suppose $F$ is a weakly usco map from $T$ to $X$. Then there exists a dense and $G_{\delta}$ subset $G$ of $T$ such that $F$ is a.l.s.c. at each $t \in G$.

We apply Christensen's theorem in conjunction with the following lemma.

LemMA 5.2. Let $X$ be a normed linear space, and let $C$ be a polytope in $X$. Let $c$ be a element of $C$ of minimal norm. Then for each $\alpha \in(0,1)$, the point ac is the unique element of conv $(\{\alpha c\} \cup c)$ of minimal norm.

Proof: The assertion is true if $c=\theta$. Otherwise, choose $y \in X^{*}$ separating 
$\|c\| U$ from $C$ such that

$$
\sup \{\langle y, x\rangle \mid\|x\| \leqslant\|c\|\}=\|c\|=\inf \{\langle y, x\rangle \mid x \in C\}
$$

By this choice of $y$, we have $\langle y, c\rangle=\|c\|$ and $\|y\|=1$. Fix $\alpha \in(0,1)$, and suppose $x$ is an element of conv $(\{\alpha c\} \cup c)$ other than $\alpha c$ itself. There exist points $c_{1}, c_{2}, \ldots, c_{n}$ in $C$ and nonnegative scalars $\beta \neq 1$ and $\beta_{1}, \beta_{2}, \ldots, \beta_{n}$ such that $x=\beta(\alpha c)+\sum \beta_{i} c_{i}$ and $\beta+\sum \beta_{i}=1$. If $\beta=0$, then $x \in C$ and $\|x\| \geqslant\|c\|>\alpha\|c\|=\|\alpha c\|$. Otherwise, set $c_{0}=\beta c+\sum \beta_{i} c_{i}$. Since $x=c_{0}-\beta(1-\alpha) c$ and since $c_{0} \in C$, we have

$$
\begin{aligned}
\|x\|=\|y\| \cdot\|x\| \geqslant\langle y, x\rangle & =\left\langle y, c_{0}\right\rangle-\beta(1-\alpha)\langle y, c\rangle \\
& >\|c\|-(1-\alpha)\|c\| \\
& =\alpha\|c\|=\|\alpha c\| .
\end{aligned}
$$

This proves that $\alpha c$ is the unique element of minimal norm.

Theorem 5.3. Let $X$ be a separable reflexive space. If $C(X)$ is equipped with the Mosco topology, then there exists a dense and $G_{\delta}$ subset $G$ of $X \times C(X)$ such that for each $(x, C)$ in $G$, the metric projection of $x$ onto $C$ is a singleton.

Proof: By Theorem 5.1, the metric projection $P$ from $X \times C(X)$ into $X$ is weakly usco; so, by Christensen's Theorem there exists a dense and $G_{\delta}$ subset $G$ of the product such that at each $(x, C) \in G, P$ is a.l.s.c.. We show that at each point of $G$, the metric projection is single-valued. Fix $(x, C)$ in $G$. Suppose $P\left(x, C^{\prime}\right)$ is not a singleton. Let $x_{0} \in P(x, C)$ be as in the definition of almost lower semicontinuity. Let $x_{1}$ be a different point of $P(x, C)$, and set $\varepsilon=\left\|x_{1}-x_{0}\right\| / 2$. By almost lower semicontinuity, there exist open subsets $V_{1}, V_{2}, \ldots, V_{n}$ of $X$ and a weakly compact. subset $K$ of $X$ such that

$$
\bigcap_{i=1}^{n} V_{i}^{-} \cap\left(K^{c}\right)^{+}
$$

is a neighbourhood of $C$, and whenever $z$ is sufficiently close to $x$ and $A \in C(X)$ lies in this neighbourhood, then $P(z, A) \cap\left(x_{0}+\varepsilon U\right) \neq \emptyset$. Pick $c_{i} \in C \cap V_{i}$ for $i=1,2, \ldots, n$. and let $B=\operatorname{conv}\left(\left\{x_{1}, c_{1}, c_{2}, \ldots, c_{n}\right\}\right)$. Now if $\alpha$ is chosen sufficiently close to zero, then

$$
B_{\alpha}=\operatorname{conv}\left(\left\{\alpha x+(1-\alpha) x_{1}, c_{1}, c_{2}, \ldots, c_{n}\right\}\right)
$$

fails to meet $K$, and $\left\|\alpha x+(1-\alpha) x_{1}-x_{0}\right\|>\varepsilon$. By Lemma 5.2, we have $P\left(x, B_{\alpha}\right)=$ $\left\{\alpha x+(1-\alpha) x_{1}\right\}$, so that $P\left(x, B_{\alpha}\right)$ fails to meet $x_{0}+\varepsilon U$. This violates almost lower semicontinuity, and we conclude that $P(x, C)$ is indeed a singleton for each $(x, C) \in$ $G$. 
Theонем 5.4. Let $X$ be a separable reflexive space. Then there is a dense and $G_{\delta}$ subset of $\left\langle C(X), \tau_{M}\right\rangle$ each element of which is almost Chebyshev.

PROOF: This is an immediate consequence of Theorem 5.3 and the KuratowskiUlam Theorem $[25$, p.247].

In [6], I showed that if $X$ is finite dimensional, then most closed convex sets are actually Chebyshev with respect to $\tau_{M}$. In particular, the closed and bounded strictly convex sets form a dense and $G_{\delta}$ collection of Chebyshev subsets of $C^{\prime}(X)$. By Lemma 4.2 , the polytopes are dense in $C(X)$ when $X$ is infinite dimensional; so, we might expect the same result to hold in a separable reflexive space. Unfortunately, the closed and bouncled convex sets form a set of first category with respect to $\tau_{M I}$ when $X$ is infinite dimensional. To see this, let $\mathcal{A}_{n}=\{C \in C(X) \mid C \subseteq n U\}$. Suppose $C \in \mathcal{A}_{n}$ hits each of the open sets $V_{1}, V_{2}, \ldots, V_{m}$ and misses the weakly compact set $I^{-}$. We can strongly separate each $x \in K$ from $C$; so, by the weak compactness of $K, C$ is contained in a finite intersection of closed halfspaces disjoint from $K$. Thus, there is an unbounded closed convex set that meets each $V_{i}$ and misses $K$, and we conclude that each $\mathcal{A}_{n}$ has empty interior. Now from the representation $\mathcal{A}_{n}=\left(\{x \mid\|x\|>n\}^{-}\right)^{c}$, we see that each $\mathcal{A}_{n}$ is $r_{M}$-closed. Thus, $\cup \mathcal{A}_{n}$, the collection of closed and bounded convex sets, is a countable union of nowhere dense sets.

\section{REFERENCES}

[1] H. Attouch, 'Famille d'opérateurs maximaux monotones et mésurabilité', Ann. Mat. Pura Appl. (4) 120 (1979), 35-111.

[2] H. Attouch, Variational convergence for functions and operators (Pitman Publishers, Boston, 1984).

(3) M. Baronti and P. Papini, 'Convergence of sequences of sets, in', Methods of functional analysis in approximation theory ISNM 78 (Birkhäuser Verlag, 1986).

[4] G. Beer, 'Metric spaces with nice closed balls and distance functions for closed sets', Bull Austral. Soc. 35 (1987), 81-96.

[5] G. Beer, 'On the straight line path for convex sets', Israel J. Math. 58 (1987), 205-212.

[6] G. Beer, 'Metric projections and the Fell topology', (submitted).

[7] G. Beer, 'Convergence of continuous linear functionals and their level sets', (submitted).

[8] A. Brown, 'A rotund reflexive space having a subspace of codimension two with a discontinuous metric projection', Michigan Math. J. 21 (1976), 145-151.

[0] C. Castaing and M. Valadier, Convex analysis and measurable multifunctions: Lecture notes in mathematics 580 (Springer-Verlag, Berlin, 1977).

[10] J.P.R. Christensen, 'Theorems of Namioka and R.E. Jolnson type for upper semicontinuous and compact valued set-valued mappings', Proc. Amer. Math. Soc. 86 (1982), 649-655.

[11] F. DeBlasi and J. Myjak, 'On the minimum distance to a closed convex set in a Banach space', Bull. Acad. Pol. Sci. 29 (1981), 373-376.

[12] F. De Blasi and J. Myjak, 'Weak convergence of convex sets in Banach spaces', Arch. Math. 47 (1986), 448-456. 
[13] F. Deutsch, W. Pollul and I. Singer, 'On set-valued metric projections, Hahn-Banach extension maps and spherical image maps', Duke Math. J. 40 (1973), 355-370.

[14] F. Deutsch and $P$. Kenderov, 'Continuous selections and approximate selections for set-valued mappings and applications to metric projections', SIAM J. Math. Anal 14 (1983), 185-194.

[15] J. Diestel, Geometry of Banach spaces-selected topics: Lecture notes in mathematics 485 (Springer-Verlag, New York, 1975).

[16] J. Fell, 'A. Hausdorff topology for the closed subsets of a locally compact non-Hausdorff space', Proc. Amer. Math. Soc. 13 (1962), 472-476.

[17] M. Fort, 'Points of continuity of semi-continuous functions', Publ. Math. Debrecen 2 (1951), 100-102.

[18] S. Francaviglia, A. Lechicki and S. Levi, 'Quasi-uniformization of hyperspaces and convergence of nets of semicontinuous multifunctions', J. Math. Anal. Appl (1985), 347-370.

[19] A. Garkavi, 'On Chebyshev and almost Chebyshev subspaces', Izv. Akad. Nauk SSSR Ser.Mat. 28 (1964), 799-818.(translation in Amer. Math. Soc. Transl. 96 (1970), 153-175).

[20] R. Holmes, A course in optimization and best approximation: Lecture notes in mathematics 257 (Springer-Verlag, New York, 1972).

[21] K. John and V. Zizler, 'A renorming of dual spaces', Israel J. Math. 12 (1972), 331-336.

[22] J. Joly, 'Une famille de topologies sur l'ensemble des fonctions convexes pour lesquelles la polarité est bicontinue', J. Math. Pures Appl 52 (1973), 421-441.

[23] P. Kenderov, 'Points of single valuedness of multivalued metric projections', C. R. Acad. Bulgare Sci. 20 (1976), 773-777.

[24] E. Klein and A. Thompson, Theory of correspondence (Wiley, Toronto, 1984).

[25] K. Kuratowski, Topology 1 (Academic Press, New York, 1966).

[26] S. Levi and A. Lechicki, 'Wijsman convergence in the hyperspace of a metric space', Boll. $U_{n}$ Mat. Ital 5-B (1987 pages 435-452).

[27] E. Michael, 'Topologies on spaces of subsets', Trans. Amer. Math. Soc. 71 (1951), 152-182.

[28] U. Mosco, 'Convergence of convex sets and of solutions of variational inequalities', Adv. in Math. 3 (1969), 510-585.

[29] U. Mosco, 'On the continuity of the Young-Fenchel transform', J. Math. Anal. Appl. 35 (1971), 518-535.

[30] G. Salinetti and R. Wets, 'On the relation between two types of convergence for convex functions', J. Math. Anal. Appl. 60 (1977), 211-226.

[31] G. Salinetti and R. Wets, 'On the convergence of sequences of convex sets in finite dimensions', SIAM Rev. 21 (1979), 18-33.

[32] Y. Sonntag, Convergence au sens de Mosco; théorie et applications à l'approximation des solutions d'inéquations (Thèse d'Etat, Université de Provence, Marseille, 1082).

[33] A. Taylor and D. Lay, Introduction to functional analysis (Wiley, New York, 1980).

[34] T. Zolezzi, Approximazioni e perturbazioni di problemi di minimo. (in preparation).

\author{
Department of Mathematics \\ California State University, Los Angeles \\ Los Angeles, CA 90032 \\ United States of America
}

